\title{
Pearls from Medical Genetics Clinic
}

\author{
Laura C. Swanson • Virginia P. Sybert
}

Published online: 30 August 2013

(C) Springer Science+Business Media New York 2013

\begin{abstract}
A medical genetics clinic is a unique and complex environment in which care requires a combination of medical knowledge, social intelligence, and emotional sensitivity. We offer a few pearls from our medical genetics clinic that we hope will help the pediatric or general dermatologist when caring for patients with known or suspected genetic disorders. This paper addresses five main topics: When is it more than just atopic dermatitis? How should the clinician approach blistering in the newborn period? What is mosaicism? When is genetic testing useful? Where can clinician, patients, and families turn for available resources?
\end{abstract}

Keywords Genodermatosis · Atopic dermatitis · Blistering newborn $\cdot$ Mosaicism

\section{Introduction}

A medical genetics clinic is a unique and complex environment that requires a combination of medical knowledge, social intelligence, and emotional sensitivity. It invites unique and interesting patients and families, presents challenging decisionmaking opportunities, and encourages clinicians to think outside the box about the rare and unusual. We have compiled a few pearls from our experience in our Medical Genetics Clinic to help guide the pediatric or general dermatologist when seeing

L. C. Swanson $(\bowtie)$

Division of Dermatology, Department of Medicine, University of Washington Medical Center, Box 356524, Seattle, WA 98195, USA e-mail: laura125@uw.edu

\section{P. Sybert}

Group Health Permanente Division of Medical Genetics, Department of Medicine, University of Washington School of Medicine, Seattle, WA, USA patients with potential or diagnosed genetic conditions. This paper will focus on the following topics:

- When is it more than just atopic dermatitis?

- How should the clinician approach blistering in the newborn period?

- What is behind cutaneous mosaicism?

- When is genetic testing useful?

- Where can clinicians, patients, and families turn for available resources?

\section{When is it More than Just Atopic Dermatitis?}

Atopic dermatitis is the most common chronic inflammatory skin disease with prevalence rates in most high-income countries of $10-30 \%$ in children and $2-10 \%$ in adults. It often begins in infancy or childhood and is characterized by pruritus and a chronic or relapsing course. It is associated with other "atopic" conditions, including asthma and allergic rhinoconjunctivitis [1].

There are particular instances in which the clinician should consider other diagnoses when evaluating a patient with presumed atopic dermatitis, especially when it is severe and unresponsive to conventional therapies. These include:

- When there are hair abnormalities

- When there are recurrent or atypical infections

- When there is failure to thrive

- When there are concerns for a nutritional or metabolic disorder

Hair abnormalities, including fragile hair, sparse hair, and poorly growing hair, may be a clue that there is an underlying disorder causing the dermatitis. Netherton syndrome (NS) (OMIM:256500), a disorder caused by mutations in SPINK5 [2], is characterized by the triad of trichorrhexis invaginata, ichthyosis linearis circumflexa (ILC), and an atopic diathesis [3]. Affected infants and toddlers often present first with pruritic rashes indistinguishable from atopic dermatitis and 
commonly do not develop the migratory, polycyclic, erythematous plaques with distinctive double-edged scale known as ILC until later [2]. Unlike patients with atopic dermatitis, patients with NS typically have thin, fragile, slow-growing, and sparse hair. With magnification, their hair demonstrates the ball and socket appearance of trichorrhexis invaginata or bamboo hair [2]. Repeat microscopic evaluation of hairs over time, including eyebrows or eyelashes, may be necessary as these changes also may not be present early on.

The distinction between atopic dermatitis and NS is critical. NS has many associated features, including mental retardation in $10-15 \%$ of patients, short stature and failure to thrive in $1 /$ 3 , and recurrent infections in most [2]. Furthermore, the condition carries with it recurrence risks for the parents of an affected child and the need for genetic counseling. Distinction also is important in terms of treatment, in particular regarding the use of topical calcineurin inhibitors.

The use of topical tacrolimus and pimecrolimus in patients with NS is controversial. In 2001, Allen et al. described three patients with NS who were treated with $0.1 \%$ tacrolimus ointment and who were all found to have tacrolimus blood levels within or above the therapeutic range for oral tacrolimus in organ transplant recipients [4]. Patients with NS have been shown to have thin stratum corneum with disorganized lamellae and incompletely processed lipids in their epidermis. They concluded that these epidermal changes lead to skin barrier dysfunction and increased percutaneous absorption of medications. Avoidance of or use of very dilute compounded formulations of topical tacrolimus only as a short-term adjunct therapy in management of flares and monitoring of serum levels, was recommended $[4,5]$.

Since the initial reports of systemic absorption of topical calcineurin inhibitors in patients with NS, there have been multiple case reports of successful treatment with both topical tacrolimus and topical pimecrolimus with good control of skin disease and without any toxic effects. Saif et al. reported four Saudi siblings treated with topical tacrolimus and pimecrolimus with good control of their skin disease and without toxicity [6]. Beljan et al. reported successful intermittent use of topical tacrolimus for acute flares in a 10-year-old boy with NS, concluding that topical therapy with tacrolimus is an easy, flexible therapeutic option for these patients [7]. Yan et al. reported three patients with NS who were treated with pimecrolimus $1 \%$ cream twice daily, resulting in marked improvement in their cutaneous disease, with detectable but very low level systemic absorption even when applied to $50 \%$ total body surface area [8].

The need for caution in the use of topical calcineurin inhibitors in patients with NS, as opposed to the widespread accepted use of topical calcineurin inhibitors in patients with atopic dermatitis, underscores the importance of distinguishing these two diseases. Netherton syndrome is a diagnosis easily missed, as the classic changes of ILC and the signature hair findings may not develop for several years.
The trichothiodystrophies (TTD) (OMIM:many) is a group of autosomal recessive disorders that share in common brittle, sulfur-deficient hair- distinguished by the type of associated multisystem abnormalities [2]. One review of 112 patients with TTD found that $65 \%$ of patients had ichthyosis [9]. These skin changes often can be mistaken for atopic dermatitis. The distinction is imperative, because TTD carries a very different prognosis and can be associated with developmental delay, short stature, ocular abnormalities, infections, photosensitivity, and defective DNA repair [9]. With TTD, the hair often is thin, brittle, fragile, and sparse. On microscopy, it can show trichoschisis and trichorrhexis nodosa-like fraying. Under polarizing microscopy, the hair shafts show the pathognomonic striking alternating dark and light (tiger tail) banding pattern [9].

Other conditions with hair shaft abnormalities and atopic dermatitis include the metabolic disorders discussed below. When suspecting a disorder with hair fragility, hairs should be cut, not plucked. The traction of plucking may cause the hairs to fracture at the weak sites, causing the structural changes to be obscured.

In patients with severe atopic dermatitis and recurrent or atypical infections, several disorders must be considered, including Wiskott-Aldrich syndrome (WAS) (OMIM:30100), hyperimmunoglobulin E syndrome (HIES) (OMIM:147060, 243700), and chronic granulomatous disease (CGD) (OMIM:233690, 233700, 233710, 306400). WAS is due to mutations in the $\mathrm{X}$-linked gene WASP and is characterized by the triad of eczematous dermatitis, thrombocytopenia, and recurrent infections [10]. Eczematous patches and plaques indistinguishable from atopic dermatitis usually develop within the first 6 months of life. These infants have severe immune defects characterized by elevated $\operatorname{IgA}$ and $\operatorname{IgE}$ and decreased IgM levels, with progressive deterioration in immune function. Recurrent infections, including pseudomonas otitis media and skin abscesses due to encapsulated organisms, account for more than $50 \%$ of deaths. Intracranial hemorrhages are responsible for $25 \%$ of deaths [2]. Patients also are at risk for developing leukemia and lymphoma [10].

Early recognition and distinction from atopic dermatitis is critical in order to initiate treatment promptly and to improve outcome, as well as to be able to provide accurate and relevant recurrence risks to family members. Presa et al. suggest that eczema similar to atopic dermatitis but with hemorrhagic features, which is a common skin finding in patients with WAS, is a key differentiating finding [11•].

Hyperimmunoglobulin E syndrome (HIES), is a genetically heterogeneous disorder with autosomal-dominant and autosomal recessive inheritance. The autosomal dominant condition is caused by mutations in STAT3. STAT3 protein regulates genes that are involved in cell growth and division, movement and apoptosis. This protein also transmits signals that help control the body's response to bacteria and fungi and 
is involved in the regulation of inflammation [12]. The autosomal recessive condition is due to mutations in DOCK8 and in one family appeared to be caused by mutations in TYK2 [2]. DOCK 8 encodes a family of guanine nucleotide exchange factors that interact with Rho GTPases in intracellular signaling network [13]. TYK2 is involved in cytokine pathways and also is a component of both the type I and type III interferon signaling pathways [14]. HIES is characterized by the clinical triad of elevated serum IgE levels $(>2000 \mathrm{IU} / \mathrm{mL})$, recurrent staphylococcal skin abscesses, and pneumonia with pneumatocele formation [15].

Differentiating HIES from severe atopic dermatitis can be difficult, because serum IgE levels in atopic dermatitis also may be significantly elevated [15]. Joshi et al. studied 70 patients with IgE levels $>2000 \mathrm{IU} / \mathrm{mL}$ and found that $77 \%$ had atopic dermatitis and $90 \%$ did not have HIES [16]. They found a significant positive association between $\operatorname{IgE}$ levels and the severity of eczema, but no correlation between IgE levels and the diagnosis of HIES. They concluded that in the absence of typical clinical features of HIES, elevated serum IgE levels were not predictive of HIES [16].

Clinical features suggestive of HIES include "cold" abscesses (subcutaneous staphylococcal abscesses with no evidence of inflammation), recurrent pneumonia, coarse facies, growth retardation, hidradenitis suppurativa, paronychia, and candidal infections [15]. None of these are features of atopic dermatitis and should be a clue to an alternate diagnosis.

Chronic granulomatous disease (OMIM:many) is caused by a defective NADPH oxidase, leading to phagocytic cells that cannot produce superoxidase anions necessary for bactericidal and fungicidal activity. The majority of cases are Xlinked recessive, due to mutations in $C Y B B$, and the remaining cases are autosomal recessive caused by mutations in $N C F 1, N C F 2, N C F 4$, or CYBA. All of these genes code for subunits of the NADPH oxidase complex [17]. Almost half of affected individuals present with dermatitis that can be difficult to distinguish from atopic dermatitis [2]. Individuals with CGD go on to develop recurrent suppurative skin infections, draining abscesses, lymphadenopathy, hepatosplenomegaly, failure to thrive, chronic diarrhea, and anemia, not commonly seen in atopic dermatitis, and should alert the clinician that an investigation for an underlying primary immunodeficiency is warranted. Early recognition and treatment is associated with reduced morbidity [17].

The Jeffrey Modell Foundation, a patient support group, has put together a list of ten warning signs (Table 1) to assist in the recognition of children with primary immunodeficiencies [18].

Infants and young children with atopic dermatitis in whom failure to thrive and/or signs/symptoms suggestive of a nutritional deficiency develop, may have an underlying metabolic disorder. Several entities must be considered, including acrodermatitis enteropathica (OMIM:201100), biotinidase deficiency (OMIM:253260), and Hartnup disorder (OMIM:234500).
Table 1 The 10 warning signs of primary immunodeficiency, from Jeffrey Modell Foundation [18]

1. $\geq 4$ new ear infections within 1 year

2. $\geq 2$ serious sinus infections within 1 year

3. $\geq 2$ months of oral antibiotic treatment with little effect

4. $\geq 2$ episodes of pneumonia within 1 year

5. Failure to gain weight or grow normally

6. Recurrent deep skin or organ abscesses

7. Persistent oral thrush or fungal infections on skin

8. Need for intravenous antibiotics to clear infections

9 . $\geq 2$ deep-seated infections, including septicemia

10. A family history of primary immunodeficiency

These warning signs were developed by the Jeffrey Modell Foundation Medical Advisory Board. Consultation with Primary Immunodeficiency experts is strongly suggested. (C) Jeffrey Modell Foundation

Acrodermatitis enteropathica can be due to acquired zinc deficiency but also is an autosomal recessive disorder caused by a reduction in the duodenal and jejunal absorption of zinc due to mutations in SLC 394A, a gene that codes for a zinc-transporter protein [2]. The classic cutaneous features include eczematous pink scaly plaques or erosions over the extremities, anogenital, and periorificial areas [19]. There may be softening of the nails and nail dystrophy. The hair is thin, brittle, and fragile and may show trichorrhexis nodosa and total alopecia also is common [2], harking back to the mantra that abnormal hair in atopic dermatitis most often is a sign of an underlying distinct disorder. Associated abnormalities include neurologic deficits, growth retardation, failure to thrive, and diarrhea. Recurrent infections, eye involvement, and hypogonadism have all been reported [2]. The distinction from atopic dermatitis is essential, because acrodermatitis enteropathica is easily treatable with zinc supplementation. Clues to the diagnosis include severe involvement of the diaper area, classically spared in atopic dermatitis, sudden appearance of the dermatitis days to weeks after weaning in breast-fed infants or within days of birth in formula fed infants, and failure to thrive, all of which are not characteristics of atopic dermatitis.

Biotinidase deficiency is a rare inborn error of metabolism inherited in an autosomal-recessive fashion caused by an inability to cleave biotin from other compounds due to mutations in BTD [2]. Cutaneous findings include eczematous rashes, seborrheic dermatitis, and alopecia [20]. Other characteristics of the disease include failure to thrive, neurologic abnormalities, hearing loss, laryngeal stridor, and eye findings. A prompt diagnosis is imperative, as treatment is inexpensive and prevents irreversible long-term complications, including developmental delay, hearing loss, and optic atrophy [20]. Clues that suggest more than just atopic dermatitis are the presence of failure to thrive and neurologic abnormalities.

Hartnup disorder is an autosomal-recessive disorder caused by mutations in SLC6A19, which encodes a transporter that mediates epithelial uptake of neutral amino acids in the 
kidneys and intestines leading to hyperamionaciduria when it is defective [21]. Most affected individuals in high-income countries remain asymptomatic, but when clinical features develop, the most common finding is a "pellagra-like" dermatitis that initially resembles sunburn and becomes scaly and pruritic, often resembling chronic eczema [21]. There can be associated intermittent ataxia, nystagmus, tremors, and psychiatric disturbances. Features that should help the clinician differentiate this condition from atopic dermatitis include photosensitivity and neurologic signs.

When caring for a child with presumed atopic dermatitis, the clinician may need to broaden the differential diagnosis when there is severe disease unresponsive to typical therapies, hair abnormalities, recurrent or abnormal infections, and/or failure to thrive. The family history should always be the first step on the road to diagnosis.

\section{How Should the Clinician Approach Blistering in the Newborn?}

The newborn infant with blistering at birth presents a diagnostic dilemma. The differential diagnosis is broad and includes common and benign self-limited conditions, as well as progressive and fatal conditions. Common self-limited considerations include sucking blisters and birth-trauma-induced blisters. Infectious causes include bullous impetigo, staphylococcal scaled skin syndrome, neonatal candidiasis, herpes simplex, and varicella. Autoimmune bullous disorders to consider include bullous pemphigoid, pemphigoid gestationis, and pemphigus vulgaris. Bullous mastocytosis and Stevens Johnson syndrome/toxic epidermal necrolysis also may present in the newborn [22]. Genetic blistering disorders that can present in the newborn include all subtypes of epidermolysis bullosa (EB) (OMIM:many), incontinentia pigmenti (OMIM:308300), ectodermal dysplasia (OMIM:305100), epidermolytic hyperkeratosis (OMIM:113800), pachyonychia congenita (OMIM:167200, 167210, 260130), ichthyosis bullosa of Siemens (OMIM:607936), ichthyosis hystrix (OMIM:146590, 146600), and peeling skin syndrome (OMIM:270300) [22].

When evaluating a newborn with blisters, as infectious causes are by far the most common, prophylactic coverage for suspected or likely pathogens may be appropriate while the diagnostic workup proceeds. Nischler et al. published a helpful algorithm (Fig. 1) to guide clinicians when evaluating a newborn with blisters [22].

Some diagnostic considerations can be confirmed or excluded by physical examination, but for others, a skin biopsy must be performed. Decisions about the site of the biopsy, the handling of the specimen, and the specific type of analysis are critical. Routine light microscopy of a blister allows the pathologist to identify the anatomical level of the split and the nature of the inflammatory infiltrate and may be sufficient if autoimmune bullous disorders and/or EB are not suspected but provide little help for these latter diagnoses [22]. If autoimmune bullous disorder is suspected, a biopsy or perilesional skin for direct immunofluorescence (DIF) is necessary, because it will detect the immune deposits in the epidermal basement membrane zone. If an epidermolysis bullosa is suspected, routine light microscopy is rarely helpful and subject to artifact and biopsy should be sent for immunofluorescence mapping (IFM) or for transmission electron microscopy (TEM). IFM provides information as to the precise level of tissue separation and the relative expression and distribution of the various protein antigens at the basement membrane zone [23•]. IFM is now favored over transmission electron microscopy (TEM) for several reasons. IFM is more widely available, cheaper, and more sensitive and specific compared with TEM. IFM is reported to be $97 \%$ sensitive and $100 \%$ specific for diagnosing EB, whereas TEM is reported to be only $71 \%$ sensitive and $81 \%$ specific [24•]. The biopsy should be taken from the edge of a fresh blister ( $1 / 2$ blister, $1 / 2$ uninvolved skin) that is $<12$ hours old. A fresh blister should be induced by firmly applying a pencil eraser to an area of skin nearby the blistered skin and rotating it laterally back and forth until erythema appears, then waiting at least 5 minutes before taking a biopsy [23•].

The initial clinical presentation of EB is not reliably predictive of the underlying EB subtype and prognosis. An accurate and definitive diagnosis is required to provide intelligent, accurate, and informed targeted counseling.

$\mathrm{X}$-linked incontinentia pigmenti, due to mutations in $I K B K G$, also presents with blistering in the newborn. The distribution of blisters on an erythematous base along the lines of Blaschko may be sufficient for diagnosis, especially if there is a positive family history. Light microscopy may be adequate for confirmation of the diagnosis if the clinical appearance is not classic.

Perhaps an unappreciated "pearl" is the reminder that a family history and an examination of parents, especially mothers when an X-linked condition is suspected, may be pivotal to arrive at the correct diagnosis in genetic skin disorders.

\section{What is Behind Cutaneous Mosaicism?}

It is not uncommon for dermatologists to encounter patients with cutaneous lesions that follow a particular patterned distribution. We now know that many of these occurrences represent somatic mosaicism for postzygotic mutations, mutations that are not present in the egg or sperm but which have occurred spontaneously in a cell during embryogenesis and are expressed only in the daughter cells derived from that progenitor. It is helpful for the clinician to have an understanding of the molecular mechanisms behind these clinical patterns when providing counseling to patients and families. 


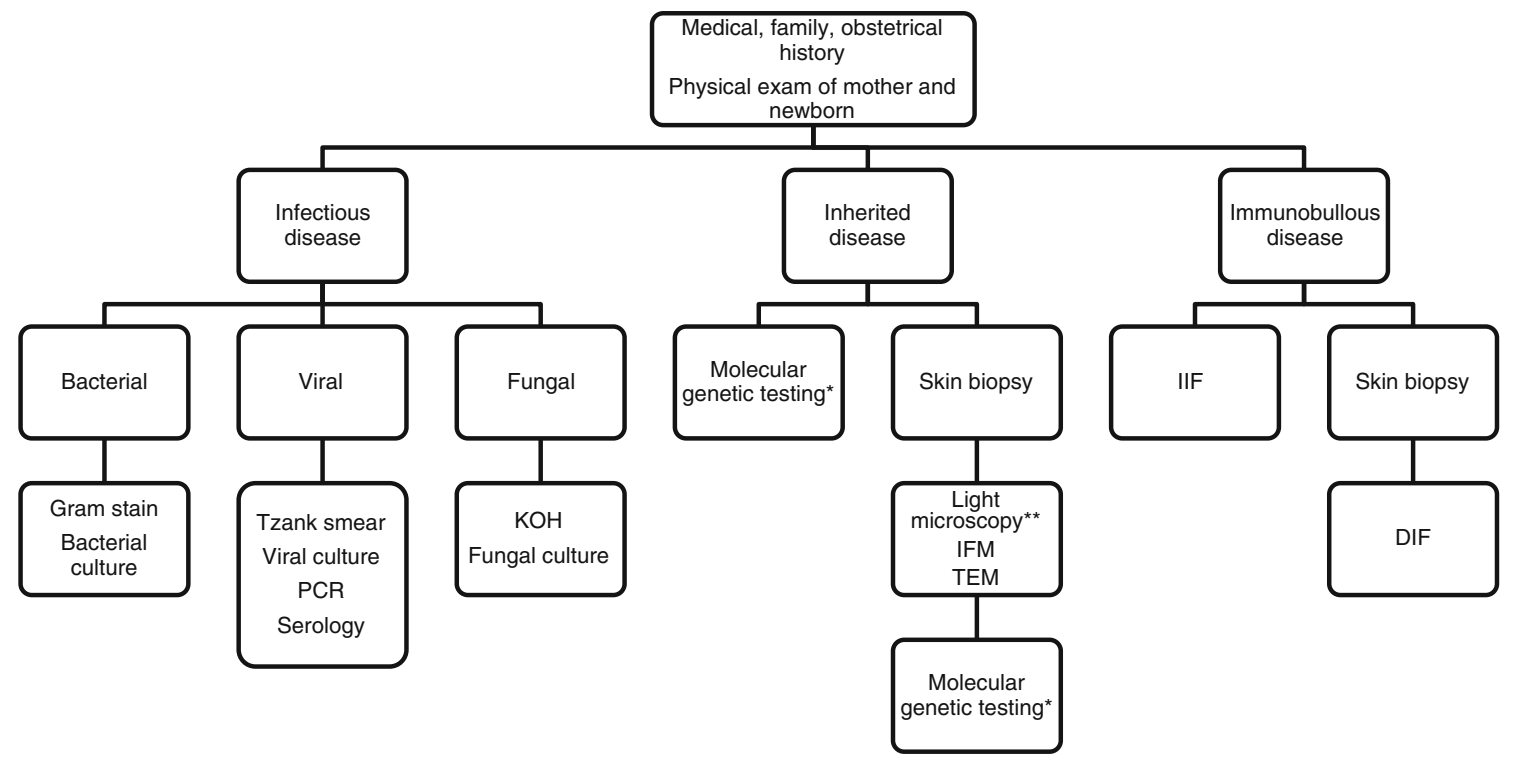

Fig. 1 Diagnostic algorithm of blisters and erosions in newborns. Modified from Nischler et al. [22]. IFM: immunofluorescence mapping; TEM: transmission electron microscopy; IIF: indirect immunofluorescence;

More than a century ago, Blaschko studied a variety of congenital and acquired skin lesions and described the characteristic lines and whorls that these skin lesions follow, now known as Blaschko's lines. Around the same time, Montgomery theorized that these lines represent the embryonic migration pathways of skin cells. However, this theory was not widely accepted until the late 1970s, when Robert Jackson published a review article on the lines of Blaschko [25]. Since that time, many of the mechanisms by which these cutaneous lesions occur have been elucidated.

The distribution and pattern of skin lesions in cutaneous mosaicism is dependent on the timing of the genetic alteration and the subtype of cells affected. Mutations that occur early in embryologic development usually affect a broader body surface area than mutations that occur later in development. Different cell types have different maturation and migration patterns that may affect the physical distribution of clinical expression in the skin. Embryonic keratinocytes move outwards from the neural crest in continuous lines and somatic mutations expressed in keratinocytes can result in cutaneous lesions following Blaschko's lines. Melanoblasts migrate individually to the skin, where they proliferate and somatic mutations occurring in melanocyte precursors can result in cutaneous lesions expressed either along Blaschko's lines or in block-like or phylloid patterns. Dermal vessels and fibroblasts take different routes, and mosaic lesions involving these tissues rarely are distributed along Blaschko's lines, but instead more often correspond to segments or dermatomes [26].

Happle classified these different patterns of expression of cutaneous mosaicism as follows: type 1, lines of Blaschko (1a narrow lines, $1 \mathrm{~b}$ broad lines); type 2, checkerboard; type 3,
DIF: direct immunofluorescence. *May not be necessary in all instances **Not appropriate if EB is highly suspected

phylloid; type 4, large patches without midline separation; and type 5, lateralization [27].

Mechanisms of mosaicism, the presence of two or more genetically or chromosomally distinct cell populations in a single individual, include postzygotic (somatic) mutations, lyonization, and revertant mosaicism. Postzygotic (somatic) mutations are caused by a mutation or chromosomal replication error that occurs after fertilization. Lyonization is the process that occurs in normal females when one X-chromosome (either maternal or paternal) in each cell is randomly inactivated during embryologic development. The inactivation remains fixed and present in all daughter cells. Thus, normal human females are functional mosaics for most, but not all, of the genes on the Xchromosome. Some cells will express the paternal alleles, others the maternal. Revertant mosaicism refers to the spontaneous in vivo correction of an inherited mutation due to mutations that occur during mitotic cell division [26].

Particular subtypes of mosaicism include gonadal mosaicism, gonosomal mosaicism, and segmental mosaicism. Gonadal mosaicism occurs when there are genetically distinct populations in the germ-line tissues only. This can explain instances when there is transmission of an autosomal dominant condition to more than one child from unaffected parents. Gonosomal mosaicism refers to cases in which mutations are present in both somatic and gonadal tissue and may result in a segmental presentation in a parent and full-blown expression in the offspring. Some individuals with somatic mosaicism will not have involvement of the germline and there will be no increased risk of transmission to offspring. Segmental mosaicism is classified into type 1 and type 2, which will be described later in detail [28]. 
Females who carry X-linked disorders are functionally mosaic due to lyonization. In X-linked dominant conditions, the mutated gene is lethal in hemizygous male embryos. Therefore, the disorder is seen exclusively in heterozygous female patients, who usually have a mosaic pattern of skin lesions. Examples of this situation include incontinentia pigmenti, Conradi-Hunermann-Happle syndrome (OMIM:302960), and focal dermal hypoplasia (Goltz syndrome) (OMIM:305600). In X-linked recessive conditions, the altered gene is not lethal in hemizygous males, and they will exhibit diffuse involvement. Heterozygous female carriers have variable manifestations dependent upon the proportion and distribution of cells in which the X-chromosome carrying the mutated gene is active. Examples include X-linked hypohidrotic ectodermal dysplasia (OMIM:305100), dyskeratosis congenita (OMIM:305000), and Menkes disease (OMIM:309400). X-inactivation patterns do not influence transmission risks, as both $\mathrm{X}$-chromosomes have an equal chance of representation in the ovaries. Conceptions to a woman with an X-linked disorder (clinically affected or not) have a $50 \%$ likelihood of inheriting either X; thus in X-linked recessive conditions there is a $25 \%$ likelihood of an affected male, an unaffected male, an unaffected female, and a carrier female - who in turn will be a carrier who will express the condition dependent upon the process of $\mathrm{X}$ inactivation in her embryogenesis. In X-linked dominant disorders, the risks are the same, but affected male conceptions will usually die in utero and affected females will show clinical findings.

There are two settings in which autosomal-dominant conditions can occur in a mosaic distribution. Type 1 segmental manifestations result from a de novo postzygotic mutation that occurs during embryonic development in one allele of a gene that is otherwise normal, resulting in features of an autosomal dominant condition in a Blaschkoiform distribution or other mosaic pattern that corresponds to the distribution of cells with that mutation. The background skin is normal. There is usually no family history of the disorder, but if the mutant cells are present in the gonads, offspring are at risk to inherit the gene for the disorder. This phenomenon has been seen in many genetic conditions; examples of disorders with cutaneous manifestations include linear/segmental Darier disease (OMIM:124200), neurofibromatosis type 1 (OMIM:162200) , and widespread epidermal nevi.

Type 2 segmental manifestations occur in an individual who has an autosomal dominant disorder in whom a postzygotic mutation inactivates or deletes the normal allele of the same gene during embryonic development. This results in more severe or earlier onset of lesions in a mosaic distribution that are superimposed on a background of milder or clinically unapparent generalized disease. Type 2 segmental mosaicism has been confirmed for Hailey-Hailey disease (OMIM:169600), Cowden syndrome (OMIM:158350), and neurofibromatosis type 1 (OMIM:162200).
Autosomal dominant lethal disorders rescued by mosaicism have been described. There are some mutations in autosomal genes that would cause early embryonic death if present in all cells and are only seen in a mosaic state. Examples include McCune-Albright syndrome (OMIM:174800), due to mutations in $G N A S 1$, and Proteus syndrome (OMIM:176920), due to mosaicism for mutations in AKT1.

\section{When is Genetic Testing Useful?}

The availability of genetic testing does not necessarily translate to utility. Molecular testing may not be appropriate for an individual or family. A clinical diagnosis may be adequate and molecular confirmation unnecessary. Molecular testing may have less than $100 \%$ sensitivity, and results can sometimes add to confusion rather than lead to clarity. Mutations may be detected that are not clearly deleterious and variants of unknown significance, alterations from the "canonical" DNA sequence of a gene that have not been seen in association with the suspected disorder and that do not appear to result in functional alteration of the gene product (based on a number of criteria including the specific amino acid change, conservation of the DNA sequence across species, etc.) may muddy the waters. Molecular diagnosis may not change medical management. Molecular testing may be indicated when a diagnosis is not possible by other means. Molecular confirmation of a suspected diagnosis may be important when the results would affect prognosis or treatment, identify asymptomatic family members at risk and for whom treatment would be possible, help delineate recurrence risks, and/or allow for prenatal diagnosis.

When pursuing molecular diagnosis, it is important to consider the cell lineages likely to be informative. In a patient in whom the deleterious gene is present in all the tissues, mutation can be detected in standard blood or buccal sample, but in a patient with a type 1 mosaic condition, DNA from affected tissues is necessary for diagnosis, as the mutation may not be found in unaffected tissues.

The availability and type of testing (karyotyping, targeted single gene analysis, comparative genome hybridization, whole exome or whole genome sequencing, deletion/duplication testing, etc.) and the cost of testing and insurance coverage may differ greatly and may be possible only on a research and not a clinical basis. A number of resources are discussed below to which the clinician can turn for information regarding genetic testing. Genetic analysis often is expensive (albeit costs are decreasing rapidly), and it is important to determine coverage by the patient's insurance, which may require prior authorization. A full explanation of the limitations of testing should be made before proceeding with testing. This can be time-consuming and it may be both in 
the physician's and the family's best interest to make a referral to a medical genetics clinic for this. Interpretation of results can be tricky and often beyond the resources of the practicing dermatologist.

\section{Where can Clinicians, Patients, and Families Turn for Available Resources?}

In counseling patients and families with known or suspected genetic conditions, the provider needs to have a familiarity with the available resources. The National Organization for Rare Disorders (NORD) is a voluntary health organization dedicated to helping people with orphan diseases and the organizations that serve them [29]. NORD provides information about rare diseases through their user-friendly website and has a Rare Disorder Database for patients and their families. It includes information about the disorder and available clinical trials and investigational products. Its Research Program provides small grants to academic scientists and it partners with pharmaceutical and biotechnology companies to ensure that certain medications are available to uninsured or under-insured individuals. It administers copay assistance and travel assistance programs. The website is http://www. rarediseases.org/.

The Genetic Alliance includes more than 1,200 diseasespecific advocacy organizations, as well as several universities, companies, government agencies, and policy organizations [30]. It offers several resources, including a web tool called "BabysFirstTest.org" to learn about newborn screening and offers a venue for families and health professionals to share their questions and experiences. "Disease InfoSearch" is an online database of advocacy organizations and resources for genetic conditions. It includes support group information and links to disease-specific materials. "Does it Run in the Family" assists users to create customized family health history materials, helping people collect, organize, and understand their family health history. "Trust it or Trash it" encourages critical thinking about health information. The website is available at http://www.geneticalliance.org/.

Registries for All (Reg4ALL) is a newly created registry platform that allows users to submit information about their health and to set privacy settings that reflect their comfort level with sharing that information [31]. Users can set preferences that allow researchers or disease advocacy organizations to reach out to them with news about clinical trials or new programs. The website is available at http://www.reg4all.org.

ClinicalTrials.gov provides access to clinical studies in humans on a wide variety of diseases and conditions [32]. The website is maintained by the National Library of Medicine and the NIH. Each record presents summary information about a study protocol and includes: the disease or condition; the intervention; the title, description, and design of the study; the requirements for participation; the locations where the study is being conducted; the contact information for the study locations; and links to relevant information on other health web sites.

Skin Advocate is a free smart phone app designed to help connect patients with dermatologic conditions with patient advocacy groups who can provide resources and support for them [33]. It can be downloaded at http://www.skinadvocateapp.com/.

The Genetic Testing Registry and GeneTests provide information about both research and clinical molecular testing [34]. The listing of laboratories offering testing is voluntary and may not be complete. The GeneTests website also provides information on patient/family support groups and a directory of clinics that deliver genetic counseling and prenatal/ preimplantation diagnosis services. The original Gene Tests organization, before being subsumed by the NIH, can be found at http://www.genetests.org and it is currently undergoing renovation. If testing for a particular disorder cannot be found, it can be helpful to search the literature, usually through PubMed, to identify possible sources of testing, e.g., laboratories pursuing research in the disorder may be interested in helping or know of other resources. The availability and nature of clinical molecular testing changes daily and, again, it may be appropriate to consult with a medical genetics facility to negotiate the maze.

GeneReviews offers expert-authored, peer-reviewed disease descriptions presented in a standardized format and focused on clinically relevant and medically actionable information on the diagnosis, management, and genetic counseling of patients and families with specific inherited conditions [35]. It is a great resource for identifying the specificity and sensitivity of testing. It is available at http://www.ncbi.nlm.nih.gov/books/NBK1116/.

\section{Conclusions}

Inherited skin disorders are rare individually, but in the aggregate present frequently enough to challenge the dermatologist and pediatrician. In the molecular era, the need for accurate diagnosis, appropriate testing, and relevant counseling is more important than ever. The landscape is changing rapidly; we hope this information, and the resources provided, will serve to keep practitioners in the swim with their heads above water in the gene pool.

Conflict of Interest LC Swanson declares no conflicts of interest. VP Sybert declares no conflicts of interest.

Human and Animal Rights and Informed Consent This article does not contain any studies with human or animal subjects performed by any of the authors. 


\section{References}

Papers of particular interest, published recently, have been highlighted as:

- Of importance

- Of major importance

1. Bieber T, Bussmann C. Atopic dermatitis. In: Bolognia JL, Jorizzo JL, Schaffer JV, et al., editors. Dermatology. Connecticut: Elsevier Saunders; 2012. p. 203-19.

2. Sybert VP. Genetic Skin Disorders. New York: Oxford University Press; 2010.

3. Sun JD, Linden KG. Netherton syndrome: a case report and review of the literature. Int J Dermatol. 2006;45:693-79.

4. Allen A, Siegfried E, Silverman R, et al. Significant absorption of topical tacrolimus in 3 patients with Netherton syndrome. Arch Dermatol. 2001;137(6):747-50.

5. Shah KN, Yan AC. Low but detectable serum levels of tacrolimus seen with the use of very dilute, extemporaneously compounded formulations of tacrolimus ointment in the treatment of patients with Netherton syndrome. Arch Dermatol. 2006;142(10):1362-3.

6. Saif GB, Al-Khenaizan S. Netherton syndrome: successful use of topical tacrolimus and pimecrolimus in four siblings. Int J Dermatol. 2007;46:290-4.

7. Beljan G, Traupe H, Metze D, et al. Comel-Netherton syndrome with bacterial superinfection. Hautarzt. 2003;54(12):1198-202.

8. Yan AC, Honig PJ, Ming ME, et al. The safety and efficacy of pimecrolimus, $1 \%$, cream for the treatment of Netherton syndrome: results from an exploratory study. Arch Dermatol. 2010;146(1):57-62.

9. Faghri S, Tamura D, Kraemer KH, et al. Trichothiodystrophy: a systematic review of 112 published cases characterizes a wide spectrum of clinical manifestations. J Med Genet. 2008;45(10):609-21.

10. Epps RE. Atopic dermatitis and ichthyosis. Pediatr Rev. 2010;31(7):278-86.

11. - Presa JG, Carvalho VO, Morrisey LR, et al. Cutaneous manifestations in patients with Wiskott-Aldrich syndrome submitted to hematopoietic stem cell transplantation. Arch Dis Child. 2013;98:304-7. The authors evaluated the frequency of cutaneous manifestations in 24 patients with WAS and found that the most frequent cutaneous manifestation was eczema similar to atopic dermatitis (seen in $71 \%$ of patients), followed by petechiae (seen in $58 \%$ of patients), and cutaneous infections (seen in $17 \%$ of patients). Forty-six percent of patients demonstrated the classic triad of recurrent infections, thrombocytopenia, and atopic dermatitis-like lesions.

12. Genetics Home Reference: Your guide to understanding genetic conditions. Available at http://ghr.nlm.nih.gov/gene/STAT3. Accessed June 2013.

13. Genetics Home Reference: Your guide to understanding genetic conditions. Available at http://ghr.nlm.nih.gov/gene/DOCK8. Accessed June 2013.

14. Genetics Home Reference: Your guide to understanding genetic conditions. Available at http://ghr.nlm.nih.gov/gene/TYK2. Accessed June 2013.

15. Ohameje NU, Loveless JW, Saini S. Atopic dermatitis or hyper-IgE syndrome? Allergy Asthma Proc. 2006;27(3):189-291.
16. Joshi AY, Iyer VN, Boyce $T G$, et al. Elevated serum immunoglobulin $E$ (IgE): when to suspect hyper-IgE syndrome-A 10-year pediatric tertiary care center experience. Allergy Asthma Proc. 2009;30(1):23-7.

17. Low LC, Manson AL, Catron HJ, et al. Autosomal recessive chronic granulomatous disease presenting with cutaneous dermatoses and ocular infection. Clin Exp Dermatol. 2013;38(3):270-3.

18. Primary Immunodeficiency Resource Center presented by Jeffrey Modell Foundation: 10 warning signs of primary immunodeficiency. Available at http://www.info4pi.org/aboutPI/index.cfm?section= aboutPI\&content $=$ warningsigns $\&$ CFID $=3975604 \&$ CFTOKEN $=$ c593eab52e12f9f0-19ED863A-98E4-DDD2-1A4BCF17A62EBE66. Accessed June 2013.

19. Maverakis E, Fung MA, Lynch PJ, et al. Acrodermatitis enteropathica and an overview of zinc metabolism. J Am Acad Dermatol. 2007;56(1):116-24.

20. Rajendiran A, Sampath S. Biotinidase deficiency-clinching the diagnosis rapidly can make all the difference!. BMJ Case Rep. 2011.

21. Burton BK. Other genodermatoses: Enzyme deficiency diseases. In: Bolognia JL, Jorizzo JL, Schaffer JV, et al., editors. Dermatology. Connecticut: Elsevier Saunders; 2012. p. 970-5.

22. Nischler E, Klausegger A, Huttner C, et al. Diagnostic pitfalls in newborns and babies with blisters and erosions. Dermatol Res Pract. 2009;320403.

23. - Gonzalez ME. Evaluation and treatment of the newborn with epidermolysis bullosa. Semin Perinatol. 2013;37(1):32-9. This review discusses the evaluation of a newborn with a blistering disorder, including diagnosis and management. It also reviews the four major subtypes of EB.

24. - Intong LR, Murrell DF. Inherited epidermolysis bullosa: new diagnostic criteria and classification. Clin Dermatol. 2012;30(1):70-7. This paper summarizes the most recent classification of EB and discusses diagnostic techniques and clinical characteristics of each subtype.

25. Jackson R. The lines of Blaschko: a review and reconsideration: observations of the cause of certain unusual linear conditions of the skin. Br J Dermatol. 1976;95(4):349-60.

26. Moss C. Mosaicism and Linear Lesions. In: Bolognia JL, Jorizzo JL, Schaffer JV, et al., editors. Dermatology. Connecticut: Elsevier Saunders; 2012. p. 943-63.

27. Happle R. Mosicism in human skin understanding the patterns and mechanisms. Arch Dermatol. 1993;129(11):1460-70.

28. Molho-Pessach V, Schaffer JV. Blaschko lines and other patterns of cutaneous mosaicism. Clin Dermatol. 2011;29(2):205-25. This paper reviews the clinicopathologic spectrum of skin lesions that follow Blaschko's lines and discusses other types of mosaicism.

29. National Organization for Rare Disorders, Inc. Available at http:// www.rarediseases.org/. Accessed May 2013.

30. Genetic Alliance, Inc. Available at http://www.geneticalliance.org/. Accessed May 2013.

31. Genetic Alliance, Inc. Available at http://www.reg4all.org. Accessed May 2013.

32. Clinical Trials.gov: A service of the U.S. National Institutes of Health. Available at http://clinicaltrials.gov/. Accessed June 2013.

33. Skin Advocate. Available at http://www.skinadvocateapp.com/. Accessed May 2013.

34. GeneTests. Available at http://www.genetests.org. Accessed June 2013.

35. Gene Reviews. Available at http://www.ncbi.nlm.nih.gov/books/ NBK1116/. Accessed June 2013. 